\title{
Navigational Protocols of Formation Control for Multiagent Network with and without Nonlinear Dynamics
}

\author{
Shaolin Li $\mathbb{D}^{1},{ }^{1}$ Yinghui He $\mathbb{D}^{1},{ }^{1}$ and Lili Zhang $\mathbb{D}^{2}$ \\ ${ }^{1}$ Department of Applied Mathematics, Honghe University, Mengzi, Yunnan 661199, China \\ ${ }^{2}$ Department of Physics, Honghe University, Mengzi, Yunnan 661199, China \\ Correspondence should be addressed to Yinghui He; heyinghui07@163.com
}

Received 2 May 2018; Revised 9 September 2018; Accepted 17 September 2018; Published 3 October 2018

Academic Editor: Manuel De la Sen

Copyright (c) 2018 Shaolin Li et al. This is an open access article distributed under the Creative Commons Attribution License, which permits unrestricted use, distribution, and reproduction in any medium, provided the original work is properly cited.

Formation control problem for multiagent networks is investigated under the framework of leader-follower consensus. By utilizing the Lyapunov stability theory, two navigational protocols for multiagent network without and with nonlinear dynamics are derived to realize formation control, respectively. In order to achieve the expected formation, controller is adopted to each agent, and the design philosophies of control protocol are required to follow two rules: (i) the destinations of agent are required to be identified and communicating with each other through the network; (ii) at least one agent is needed to be navigator which can detect the difference between its current location and destination. Finally, the two numerical examples are provided to demonstrate the effectiveness of the proposed navigational protocols.

\section{Introduction}

Multiagent dynamical network $[1,2]$ had received extensive attention in the past two decades due to its ubiquitousness in nature and human society. The rapid development of multiagent dynamical network has been witnessed in many fields of mathematics, physics, computer science, engineering, and so on [3-6]. In a multiagent network, numerous agents are interconnected by sharing mutually their local information and achieve cooperatively the common goal. The investigations of collective behavior for multiagent dynamical network, exactly as consensus computation [4-6], synchronization [7], distributed processing [8], and network flow control $[9,10]$, have been employed widely in the fields of aerospace, robot, wireless communication, etc.

Consensus problem is one of the most profound issues on the multiagent dynamical network because of its notability in many scientific areas. In [3], authors suggested that consensus means to reach an agreement regarding a certain quantity of interest that depends on the state of all agents. A consensus algorithm (or protocol) is an interaction rule that specifies the information exchange between an agent and all of its neighbors on the network. There is an interesting and challenging theme on the consensus of a multiagent system, that is, to design simple distributed control law for each agent, using only local information from its neighbors, such that the whole network's agents can achieve prescribed consensus. The great developments are attributed to researchers and their remarkable works; lots of the innovative consensus protocols and control strategies are proposed and improved to meet the practical application, such as the distributed observerbased protocols [11, 12], output feedback protocol [13-15], event-triggered control $[8,16]$, and tracking control [1719]. It is pointed out that other characteristic topics on the consensus of the multiagent system, such as maintaining a formation [20, 21], swarming [22], and rendezvousing [23], are still worth to be investigated as a result of their practical applications.

With respect of the research topics discussed in the multiagent dynamical networks, formation control focuses on the feasibility problem of achieving a specified formation among a group of agents by distributed control and each agent relies only on locally available information, on which lots of interesting works have been reported [21, 24, 25]. In a real application, it is well known that the leader-follower formation control $[26,27]$ is employed usually to guarantee 
the achievement of prearranged formation, where the leader is a particular agent acting as an external input to steer the other agents. On the other hand, pinning control [28] is always applied to achieve synchronization in the complex networks. To some extent, there is something in common between leader-follower control and pinning control.

By taking the above considerations into account, differing from the previous work in the existing literature and based on the ideas of leader-follower control and pinning control, two navigational protocols of formation control are designed from a mathematical perspective, where agents with or without nonlinear dynamics are investigated in this paper.

The main contributions of this paper can be summarized as the following three aspects: to realize the expect formation control of a multiagent network, we can conclude that (i) the destination's information of each agent should be communicated through network; (ii) At least an agent must be chosen as navigator guiding the rest agents to reach their location, whose responsibility is to detect timely the difference between its expect state and current state; and (iii) in the case of multiagent network with nonlinear dynamics, it is necessary that each agent's nonlinear state is estimated at its destination.

The above discussions can be used to design the navigational control protocol, which conform to subjective intuition in our real life. The rest of this paper is organized as follows. Preliminaries and model description are described briefly in Section 2. In Section 3, two navigational protocols for a multiagent network without and with nonlinear dynamic are provided, where one of the agents is selected as the navigator and the associated requirements are proposed to design controllers. In Section 4, two numerical examples are designed to validate the effectiveness of navigational protocols. Finally, conclusion is drawn in Section 5.

\section{Preliminaries and Model Description}

In this section, some basic concepts of consensus, formation control, and notations of algebraic graph theory are briefly introduced. More details can be found in Godsil and Royle's work [29].

Let $G=(V, E, A)$ be a weighted undirected graph of order $N$ which consists of a node set $V=\left\{v_{1}, v_{2}, \cdots, v_{N}\right\}$, an undirect edges set $E \subseteq V \times V$, and a weighted adjacency matrix $A=\left(a_{i j}\right)_{N \times N} \in R^{N \times N}$ with $a_{i i}=0$ and nonnegative elements. An undirected edge $e_{i j}$ of $G$ is denoted by the unordered pair of nodes $\left(v_{i}, v_{j}\right)$, which means that nodes $v_{j}$ and $v_{j}$ can exchange mutually their information. Throughout this paper, the undirected graph with positive weights is considered, which shows that the communications among agents are bidirectional. Therefore, the element $a_{i j}$ of adjacency matrix $A$ satisfies the following properties: (i) $a_{i j}=a_{j i}$; (ii) $a_{i j}>0$ if and only if there is an edge between node $v_{i}$ and node $v_{j}$; otherwise, $a_{i j}=0$. According to graph theory, a path between nodes $v_{i}$ and $v_{j}$ is a sequence of edges $\left(v_{i}, v_{i_{1}}\right),\left(v_{i_{1}}, v_{i_{2}}\right), \cdots,\left(v_{i_{s}}, v_{j}\right)$, in which nodes $v_{i_{k}}(k=$ $1,2, \cdots, s)$ are distinct completely. A graph $G$ is connected if there is a path between any two nodes in $G$. It is proved that a connect network is totally equivalent to an irreducible matrix. Furthermore, the Laplacian matrix $L=\left(l_{i j}\right)_{N \times N}$ corresponding to adjacency matrix $A$ is defined by

$$
l_{i i}=-\sum_{j=1, j \neq i}^{N} l_{i j}, \quad l_{i j}=a_{i j}, i \neq j
$$

which implies the dissipative property that $\sum_{j=1}^{N} l_{i j}=0, i=$ $1,2, \cdots, N$. For any connected network, the following several lemmas [30] are employed to study the pinning synchronization in the field of complex network.

Lemma 1. If $L=\left(l_{i j}\right)_{N \times N}$ is irreducible and symmetric, for $l_{i j}=l_{j i} \geq 0$ for $i \neq j$, and $\sum_{j=1}^{N} l_{i j}=0$ for all $i=1,2, \cdots, N$, then all eigenvalues of the matrix $L-D$ are negative, where $D=\operatorname{diag}\{d, 0, \cdots, 0\} \in R^{N \times N}$ and $d$ is any positive constant.

Lemma 2. The Laplacian matrix L of an undirected network is symmetric and positive semi-definite. Moreover, L has a simple eigenvalue of 0 and the rest of the eigenvalues are negative if and only if the undirected network is connected.

Generally, the information states with agent dynamics are given by

$$
\dot{x}_{i}(t)=h_{i}, \quad i=1,2, \cdots, N
$$

where $x_{i}(t)=\left(x_{i 1}(t), x_{i 2}(t), \cdots, x_{i n}(t)\right)^{\mathrm{T}} \in R^{n}$ is state vector of the $i$ th agent and $h_{i} \in R^{n}$ is the control input. In the most existing literatures, the protocol to realize consensus with respect to the states of $N$ integrator agents is expressed as

$$
\dot{x}_{i}(t)=\sigma \sum_{j=1, j \neq i}^{N} g_{i j}\left(x_{j}(t)-x_{i}(t)\right), \quad i=1,2, \cdots, N,
$$

where $\sigma>0$ is coupling strength and $G=\left(g_{i j}\right)_{N \times N}$ is the adjacency matrix of the associated communication graph. Using the Laplacian matrix $L$ defined by (1), system (3) can be equivalently rewritten as follows:

$$
\dot{x}_{i}(t)=\sigma \sum_{j=1}^{N} l_{i j} x_{j}(t), \quad i=1,2, \cdots, N
$$

As a classical model, system (4) has been used to investigate originally the consensus for multiagent system. Furthermore, if each agent is considered as oscillator which can be described by $\dot{x}_{i}(t)=f\left(x_{i}(t)\right)$, then system (4) is modified to the following dynamical multiagent system,

$$
\dot{x}_{i}(t)=f\left(x_{i}(t)\right)+\sigma \sum_{j=1}^{N} l_{i j} x_{j}(t), \quad i=1,2, \cdots, N
$$

where $f: R^{n} \longrightarrow R^{n}$ is a continuously differentiable vector function. In the following, on the basis of models (4) and (5), the formation controls of multiagent system with linear and nonlinear dynamics are considered according to consensus principle of multiagent network. 
Definition 3. For the given initial state $x_{i}\left(t_{0}\right)=\left(x_{i 1}^{0}, x_{i 2}^{0}, \cdots\right.$, $\left.x_{i n}^{0}\right)^{\mathrm{T}} \in R^{n}$ of each agent and its target state $\alpha_{i}=\left(a_{i 1}, a_{i 2}, \cdots\right.$, $\left.a_{i n}\right)^{\mathrm{T}} \in R^{n}$, the expected formation of multiagent system (4) or (5) is achieved if

$$
\lim _{t \longrightarrow+\infty}\left\|x_{i}(t)-\alpha_{i}\right\|=0, \quad i=1,2, \cdots, N,
$$

where $\|\cdot\|$ denotes the Euclidean norm in $R^{n}$.

Next, to realize the expected formation of multiagent system (4) and (5), some input controllers will be introduced to agents. Thus, the controlled multiagent network can be derived as follows

$$
\begin{array}{r}
\dot{x}_{i}(t)=f\left(x_{i}(t)\right)+\sigma \sum_{j=1}^{N} l_{i j} x_{j}(t)+u_{i}(t) \\
\\
\quad i=1,2, \cdots, N,
\end{array}
$$

where $u_{i}(t)$ is input controller to be designed further and nonlinear function $f$ is always equal to zero or not that describes the situation of the multiagent network (7) with or without nonlinear dynamics, respectively. Particularly, an agent is chosen as a leader that guides the rest of the agents to arrive at their destination. Without loss of generality, the first agent is chosen as leader of system.

Throughout the rest of paper, the following assumptions are needed.

Assumption 4. The nonlinear function $f$ satisfies the socalled QUAD condition; that is, there exists a constant matrix $K$ such that

$$
\begin{array}{r}
(x-y)^{\mathrm{T}}(f(x)-f(y)) \leq(x-y)^{\mathrm{T}} K(x-y), \\
\forall x, y \in R^{n},
\end{array}
$$

especially, denoting that $\theta=\lambda_{\max }\left(\left(K+K^{\mathrm{T}}\right) / 2\right)$.

Assumption 5. The network (4) and (5) are connected.

It should be noted that Assumption 4 has a wide application prospect; all linear and piecewise linear functions have been proven to satisfy this condition. Furthermore, if $\partial f_{i} / \partial x_{j}(i, j=1,2, \cdots, n)$ are bounded, then the above condition is also satisfied. Therefore, some well-known systems (such as the Lorzen system, Chen system, Lü system, Chua circuit, and recurrent neural networks) can be involved.

\section{Main Results}

In this section, by virtue of designs of input controllers $u_{i}(t)$ (especially concerning the first agent), two navigational protocols are established to ensure the formation control of multiagent systems with and without nonlinear dynamics, respectively.

3.1. Navigational Protocol without Nonlinear Dynamics. First of all, we consider the formation control of multiagent network without nonlinear dynamics by using the following result.
Theorem 6. Suppose that Assumption 5 holds. The expected formation of controlled multiagent network (7) without nonlinear dynamics can be realized if the controlled protocol $u_{i}(t)$ is designed as

$$
\begin{aligned}
& u_{1}(t)=-\sigma d\left(x_{1}(t)-\alpha_{1}\right)-\sigma \sum_{j=1}^{N} l_{1 j} \alpha_{j}, \\
& u_{i}(t)=-\sigma \sum_{j=1}^{N} l_{i j} \alpha_{j}, \quad i=2,3, \cdots, N,
\end{aligned}
$$

where the feedback gain $d$ is any positive constant.

Proof. Let $e_{i}(t)=x_{i}(t)-\alpha_{i}(i=1,2, \cdots, N)$ be the error evaluating the state $x_{i}(t)$ of every agent and its target $\alpha_{i}$; the corresponding error system can be derived as

$$
\begin{aligned}
& \dot{e}_{1}(t)=\dot{x}_{1}(t)=\sigma \sum_{j=1}^{N} l_{1 j} e_{j}(t)-\sigma d e_{1}(t) \\
& \dot{e}_{i}(t)=\dot{x}_{i}(t)=\sigma \sum_{j=1}^{N} l_{i j} e_{j}(t), \quad i=2,3, \cdots, N .
\end{aligned}
$$

We denote $e(t)=\left(e_{1}^{\mathrm{T}}(t), e_{2}^{\mathrm{T}}(t), \cdots, e_{N}^{\mathrm{T}}(t)\right)^{\mathrm{T}}$, and then error system (10) can be rewritten into a compact form:

$$
\dot{e}(t)=\sigma\left((L-D) \otimes I_{n}\right) e(t),
$$

where $\otimes$ is the Kronecker product and $D=\operatorname{diag}\{d, 0, \cdots, 0\} \in$ $R^{N \times N}$

Construct the Lyapunov functional candidate:

$$
V(t)=\frac{1}{2} e^{\mathrm{T}}(t)\left(I_{N} \otimes I_{n}\right) e(t) .
$$

The derivative of $V(t)$ along the trajectories of (11) yields that

$$
\begin{aligned}
\dot{V}(t) & =e^{\mathrm{T}}(t)\left(I_{N} \otimes I_{n}\right) \dot{e}(t) \\
& =e^{\mathrm{T}}(t)\left(I_{N} \otimes I_{n}\right) \cdot\left[\sigma\left((L-D) \otimes I_{n}\right)\right] e(t) \\
& =\sigma \cdot e^{\mathrm{T}}(t)\left((L-D) \otimes I_{n}\right) e(t) .
\end{aligned}
$$

According to Lemma 1, all eigenvalues of the matrix $L-D$ are negative. Hence, $\dot{V}(t)<0$. It implies that $\lim _{t \rightarrow \infty} V(t)=$ 0 . According to the definition of $V(t)$, it is easy to see that (6) is satisfied, which indicates that the expected formation of the controlled multiagent network (7) without nonlinear dynamics is realized under the controlled protocol (9). The proof is completed.

Remark 7. According to the design of controlled protocols $u_{i}(t)$ in (9), the destination of each agent should be identified and communicating with each other through the network. Moreover, at least one agent is chosen as navigator to detect timely the difference between its current location and destination.

Remark 8. From Lemma 2, the matrix $L-D$ is always negative definite for any $d>0$. Therefore, the expected formation of multiagent network (7) without nonlinear dynamics can be realized under the designed protocol (9). 
3.2. Navigational Protocol with Nonlinear Dynamic. In the following, the above navigational protocol is generalized to the case of multiagent network with nonlinear dynamic. It should be noted that there exists nonneglected difference on the selection of feedback gain of network. Additionally, the dynamic behavior of agent is also taken into account.

Theorem 9. Suppose that Assumptions 4 and 5 hold. If there exist the suitable positive constants $\sigma, d$ such that the linear matrix inequality

$$
\theta I_{N}+\sigma(L-D)<0,
$$

is satisfied, then the expected formation of the controlled multiagent network (7) with nonlinear dynamics can be realized under the following controlled protocols,

$$
\begin{aligned}
& u_{1}(t)=-f\left(\alpha_{1}\right)-\sigma d\left(x_{1}(t)-\alpha_{1}\right)-\sigma \sum_{j=1}^{N} l_{1 j} \alpha_{j}, \\
& u_{i}(t)=-f\left(\alpha_{i}\right)-\sigma \sum_{j=1}^{N} l_{i j} \alpha_{j}, \quad i=2,3, \cdots, N,
\end{aligned}
$$

where $\theta=\lambda_{\text {max }}\left(\left(K+K^{\mathrm{T}}\right) / 2\right), D=\operatorname{diag}(d, 0, \cdots, 0) \in R^{N \times N}$.

Proof. Similar to Theorem 6, introducing the following error notations,

$$
\begin{aligned}
e_{i}(t) & =x_{i}(t)-\alpha_{i}, \\
e(t) & =\left(e_{1}^{\mathrm{T}}(t), e_{2}^{\mathrm{T}}(t), \cdots, e_{N}^{\mathrm{T}}(t)\right)^{\mathrm{T}},
\end{aligned}
$$

the error system concerning network (7) and its expected formation is derived,

$$
\begin{aligned}
\dot{e}_{i}(t)= & f\left(e_{i}(t)+\alpha_{i}\right)-f\left(\alpha_{i}\right) \\
& +\sigma \sum_{j=1}^{N} l_{i j} e_{j}(t)-\sigma d_{i} e_{i}(t), \quad i=1,2, \cdots, N,
\end{aligned}
$$

where $d_{1}=d>0, d_{i}=0, i=2,3, \cdots, N$.

Constructing the Lyapunov functional candidate

$$
V(t)=\frac{1}{2} \sum_{i=1}^{N} e_{i}^{\mathrm{T}}(t) e_{i}(t),
$$

differentiating $V(t)$ along the trajectories of (17) and using Assumption 4, one yields that

$$
\begin{aligned}
& \dot{V}(t)=\sum_{i=1}^{N} e_{i}^{\mathrm{T}}(t)\left[f\left(e_{i}(t)+\alpha_{i}\right)-f\left(\alpha_{i}\right)\right. \\
& \left.+\sigma \sum_{j=1}^{N} l_{i j} e_{j}(t)-\sigma d_{i} e_{i}(t)\right] \leq \sum_{i=1}^{N} e_{i}^{\mathrm{T}}(t)\left[K e_{i}(t)\right. \\
& \left.+\sigma \sum_{j=1}^{N} l_{i j} e_{j}(t)-\sigma d_{i} e_{i}(t)\right]=e^{\mathrm{T}}(t)\left(\left(I_{N} \otimes K\right)\right. \\
& \left.+\sigma\left((L-D) \otimes I_{n}\right)\right) e(t) .
\end{aligned}
$$

On the other hand, according to the definition of parameter $\theta$, one yields that

$$
\begin{aligned}
& \dot{V}(t) \\
& \quad \leq e^{\mathrm{T}}(t)\left(I_{N} \otimes\left(\frac{K+K^{\mathrm{T}}}{2}\right)+\sigma\left((L-D) \otimes I_{n}\right)\right) e(t) \\
& \quad \leq e^{\mathrm{T}}(t)\left(\left(\theta I_{N}+\sigma(L-D)\right) \otimes I_{n}\right) e(t) .
\end{aligned}
$$

Therefore, $\dot{V}(t)<0$ can be obtained by (14), which means $\lim _{t \rightarrow \infty}\left\|x_{i}(t)-\alpha_{i}\right\|=0$. In other words, the expected formation of the controlled multiagent network (7) is realized and it completes the proof.

Remark 10. Comparing the control protocols (9) and (15), the nonlinear state of each agent at its destination expressed by $f\left(\alpha_{i}\right)$ is introduced into the design of controller when agents of multiagent network are suffering from nonlinear dynamics. Therefore, in order to realize the expected formation control of multiagent network (7), it is reasonable to evaluate the nonlinear state of each agent at its destination. Additionally, differing from the consensus of multiagent network in which all agents achieve the same state, the formation control can be regarded as a special issue of cluster synchronization in complex network. As a result, the design of controller for formation control is more complicated than one in consensus of multiagent network.

Remark 11. For the sufficient condition (14), a convenient condition $\lambda_{\text {max }}\left(\theta I_{N}+\sigma(L-D)\right)<0$ can be applied to replace it. However, it is more conservative in real application. On the other hand, condition (14) is a typical linear matrix inequality (LMI) which can be easily solved by the LMIs toolbox of Matlab.

Remark 12. For the case of multiagent network without nonlinear dynamics, the feedback gain $d$ can be any positive constant. However, in the case of network with nonlinear dynamics, it should be pointed out that a control gain $d>0$ is suitable, which is relevant to LMI (14). On the other hand, according to adaptive technique, the following scheme can be applied to realize the formation control:

$$
\dot{d}(t)=\mu\left(x_{1}(t)-\alpha_{1}\right)^{\mathrm{T}}\left(x_{1}(t)-\alpha_{1}\right),
$$

where $\mu>0$ is an accommodation coefficient.

Remark 13. From the perspectives of physical study and real application, more elements of the formation control can be introduced into multiagent network (7), such as moment of inertia, rotation phase, and balancing adjustment. Furthermore, it is feasible that model (7) can be extended to the higher-order cases.

\section{Numerical Simulations}

In this section, two numerical examples are provided to verify the effectiveness of theoretical results proposed in Section 3. We consider a multiagent network consisting of 7 agents, 


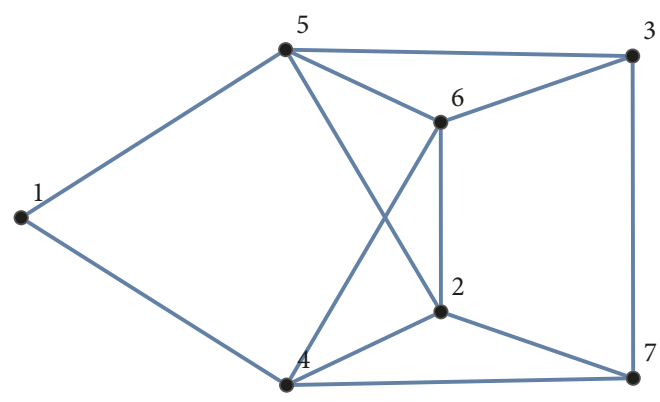

FIGURE 1: The communication topology of multiagent network, each number stands for an agent, and edge shows communication among agents.

in which the communication network is shown in Figure 1. Obviously, the network is connected.

4.1. Formation Control of Multiagent Network without Nonlinear Dynamics. In this simulation, a two-dimensional model is introduced. We consider the controlled multiagent network (7) consisting of 7 agents without nonlinear dynamics, where $x_{i}(t)=\left(x_{i 1}(t), x_{i 2}(t)\right)$, the coupling strength $\sigma=1.2$, and the corresponding Laplacian matrix $L=\left(l_{i j}\right)_{7 \times 7}$ of communication network, which is shown in Figure 1, satisfy that $l_{i j}=1$ if and only if there is an edge between distinguishing nodes $i$ and $j$; otherwise, $l_{i j}=0$. The expected formation position is given by

$$
\begin{aligned}
& \alpha_{1}=(10,4), \\
& \alpha_{2}=(10,4), \\
& \alpha_{3}=(10,-2), \\
& \alpha_{4}=(10,-4), \\
& \alpha_{5}=(12,1), \\
& \alpha_{6}=(12,-1), \\
& \alpha_{7}=(14,0) .
\end{aligned}
$$

According to Theorem 6 and without loss of generality, we choose agent 1 as navigator. Applying the navigation protocol (9), we choose the feedback gain $d=2$. In the simulation process, the initial position $x_{i}(0)$ of agents is given randomly on $[-1,1] \times[-1,1]$. It is shown in Figure 2 that the expected formation of the multiagent network is realized under the controlled protocol (9).

4.2. Formation Control of Multiagent Network with Nonlinear Dynamics. Generally, nonlinear system is more difficult to control than linear system. Here, we consider the 3dimensional multiagent network (7) that consists of 7 identical Chen oscillators, which can be described as follows:

$$
\dot{x}_{i}(t)=f\left(x_{i}(t)\right)+\sigma \sum_{j=1}^{7} l_{i j} x_{j}(t)+u_{i}(t),
$$

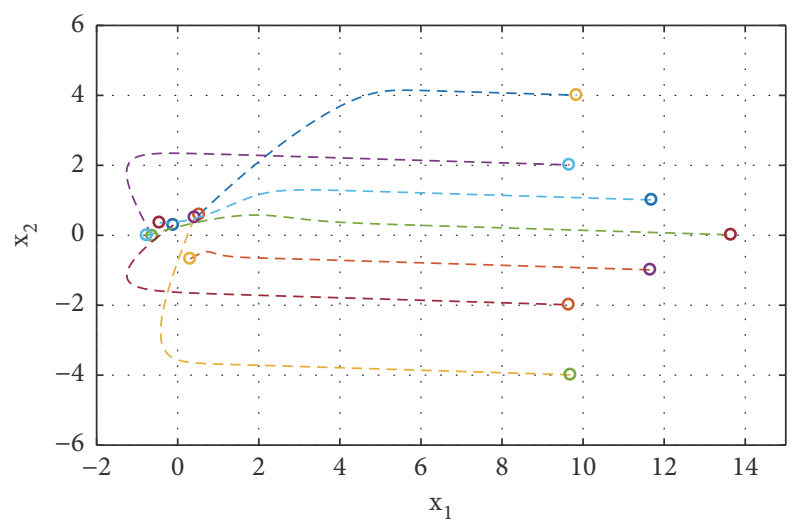

FIGURE 2: The trajectories of all agents without nonlinear dynamics between their initial positions and expected formation.

where $L=\left(l_{i j}\right)_{7 \times 7}$ is still Laplacian matrix of communication network described by Figure $1, x_{i}(t)=\left(x_{i 1}(t), x_{i 2}(t)\right.$, $\left.x_{i 3}(t)\right)^{\mathrm{T}} \in R^{3}$, and

$$
\begin{aligned}
& f\left(x_{i}(t)\right)=\left(35\left(x_{i 2}(t)-x_{i 1}(t)\right),-7 x_{i 1}(t)\right. \\
& -x_{i 1}(t) x_{i 3}(t)+28 x_{i 2}(t), x_{i 1}(t) x_{i 2}(t) \\
& \left.-3 x_{i 3}(t)\right)^{\mathrm{T}} .
\end{aligned}
$$

For the destination area $S=\left\{\left(s_{1}, s_{2}, s_{3}\right) \in R^{3}|| s_{i} \mid \leq M_{i}\right\}$ and the expected formation vector $\alpha_{i}=\left(\alpha_{i 1}, \alpha_{i 2}, \alpha_{i 3}\right)^{\mathrm{T}}$, one has

$$
\begin{aligned}
\left(x_{i}-\right. & \left.\alpha_{i}\right)^{\mathrm{T}}\left(f\left(x_{i}\right)-f\left(\alpha_{i}\right)\right) \\
= & -35 e_{i 1}^{2}+28 e_{i 1} e_{i 2}+28 e_{i 2}^{2}-3 e_{i 3}^{2}+e_{i 1} e_{i 3} \alpha_{i 2} \\
& -e_{i 1} e_{i 2} \alpha_{i 3} \\
\leq & -35 e_{i 1}^{2}+\left(28+M_{3}\right)\left|e_{i 1} e_{i 2}\right|+28 e_{i 2}^{2}-3 e_{i 3}^{2} \\
& +M_{2}\left|e_{i 1} e_{i 3}\right| \\
\leq & \left(-35+\frac{\rho\left(28+M_{3}\right)}{2}+\frac{\eta M_{2}}{2}\right) e_{i 1}^{2} \\
& +\left(28+\frac{28+M_{3}}{2 \rho}\right) e_{i 2}^{2}+\left(-3+\frac{M_{2}}{2 \eta}\right) e_{i 3}^{2} \\
\leq & \theta e_{i}^{\mathrm{T}} e_{i},
\end{aligned}
$$

where $e_{i}=\left(e_{i 1}, e_{i 2}, e_{i 3}\right)^{\mathrm{T}}=x_{i}-\alpha_{i}$. In simulation, the expected formation vectors are set as

$$
\begin{aligned}
& \alpha_{1}=(4,10,3), \\
& \alpha_{2}=(6,10,3), \\
& \alpha_{3}=(3,10,5), \\
& \alpha_{4}=(5,10,5), \\
& \alpha_{5}=(7,10,5),
\end{aligned}
$$

$$
i=1,2, \cdots, 7 \text {, }
$$




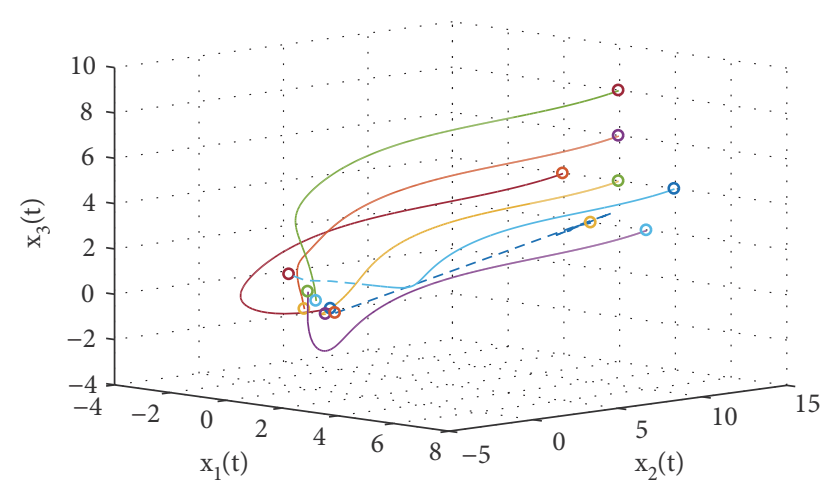

FIGURE 3: The trajectories of all agents with nonlinear dynamics between their initial formation and expected formation.

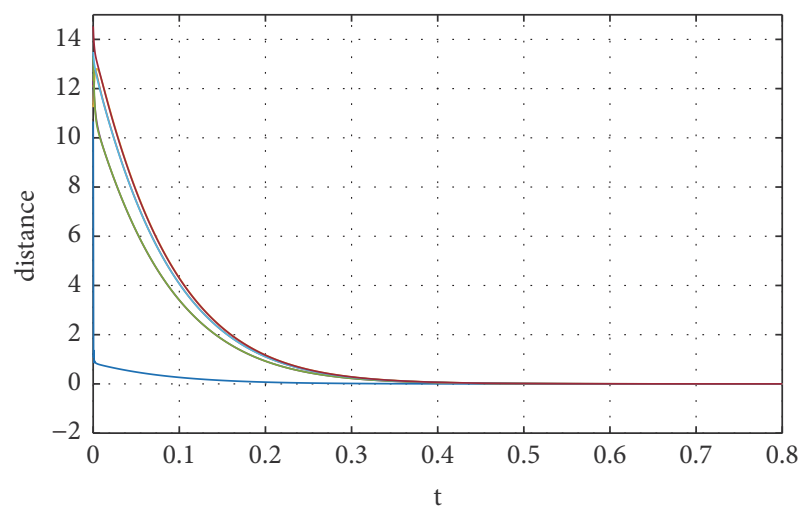

FIGURE 4: The distance of each agent between its initial position and expected position.

$$
\begin{aligned}
& \alpha_{6}=(5,10,7), \\
& \alpha_{7}=(5,10,9) .
\end{aligned}
$$

Therefore, $M_{1}=7, M_{2}=10, M_{3}=9$ can be found. Choosing $\rho=3.6425$ and $\eta=0.1386$, one obtains $\theta=$ 33.0789. Without loss of generality, agent 1 is chosen as navigator in the simulation. According to Theorem 9, the coupling strength and feedback gain are chosen as $(\sigma, d)=$ $(117,24)$, respectively. According to the above data design, LMI (14) is satisfied. The initial position $x_{i}(0)$ of each agent is given randomly on $[-1,1]^{3}$. Figure 3 shows that the expected formation of multiagent with nonlinear dynamic is realized under the controlled protocol (14) and (15). Furthermore, the distance between current position and destination of each agent as to time is presented by Figure 4, where the distance $h_{i}(t)$ is defined as $h_{i}(t)=\sqrt{\left(x_{i}(t)-\alpha_{i}\right)^{\mathrm{T}}\left(x_{i}(t)-\alpha_{i}\right)}, i=$ $1,2, \cdots, 7$. It is interesting that each agent reaches its expected destination almost at the same time.

\section{Conclusion}

In this paper, the designs of navigational protocols of formation control for multiagent network with and without nonlinear dynamics have been investigated in detail. For the case of multiagent network without nonlinear dynamics and according to navigational protocol proposed in Theorem 6 , we point out that the expected formation is always achieved for any feedback gain. Moreover, any agent can be chosen as the whole system's navigator. However, for the special case of system with nonlinear dynamics, a criterion relying on the nonlinear state at destination, the suitable coupling strength and feedback gain are proposed in Theorem 9. To achieve the expected formation control, it is worthy of concern that destination of each agent should be communicated extra through network in both cases.

Future works include investigating the simplification of controllers, input delay, and collision avoidance. From the perspective of the real application, it is important and significant to consider the heterogeneous agents due to environmental influences or implemented equipment. In addition, it is also interesting and necessary to explore the consequences of network-attacks on their communication channels.

\section{Data Availability}

The data used to support the findings of this study are available from the corresponding author upon request.

\section{Conflicts of Interest}

The authors declare that there are no conflicts of interest regarding the publication of this paper.

\section{Acknowledgments}

This work is supported by the youth academic backbone fund (No. 2014GG0105 and No. 2016GG0308) of Honghe University.

\section{References}

[1] J. Ferber, Multi-agent systems: an introduction to distributed artificial intelligence, Reading: Addison-Wesley, 1999.

[2] W. Ren, R. W. Beard, and E. M. Atkins, "A survey of consensus problems in multi-agent coordination," in Proceedings of the American Control Conference (ACC '05), Portland, Ore, USA, June 2005.

[3] R. Olfati-Saber, J. A. Fax, and R. M. Murray, "Consensus and cooperation in networked multi-agent systems," Proceedings of the IEEE, vol. 95, no. 1, pp. 215-233, 2007.

[4] W. Yu, G. Chen, and M. Cao, "Some necessary and sufficient conditions for second-order consensus in multi-agent dynamical systems," Automatica, vol. 46, no. 6, pp. 1089-1095, 2010.

[5] G. Wen, Z. Duan, Z. Li, and G. Chen, "Flocking of multiagent dynamical systems with intermittent nonlinear velocity measurements," International Journal of Robust and Nonlinear Control, vol. 22, no. 16, pp. 1790-1805, 2012.

[6] W. Yu, W. Ren, W. X. Zheng, G. Chen, and J. Lü, "Distributed control gains design for consensus in multi-agent systems with second-order nonlinear dynamics," Automatica, vol. 49, no. 7, pp. 2107-2115, 2013. 
[7] H. Zhang, F. L. Lewis, and A. Das, "Optimal design for synchronization of cooperative systems: state feedback, observer and output feedback," IEEE Transactions on Automatic Control, vol. 56, no. 8, pp. 1948-1952, 2011.

[8] D. V. Dimarogonas, E. Frazzoli, and K. H. Johansson, "Distributed event-triggered control for multi-agent systems," IEEE Transactions on Automatic Control, vol. 57, no. 5, pp. 1291-1297, 2012.

[9] R. Olfati-Saber, "Flocking for multi-agent dynamic systems: algorithms and theory," IEEE Transactions on Automatic Control, vol. 51, no. 3, pp. 401-420, 2006.

[10] Y. Fan, G. Feng, Y. Wang, and C. Song, "Distributed eventtriggered control of multi-agent systems with combinational measurements," Automatica, vol. 49, no. 2, pp. 671-675, 2013.

[11] Y. Hong, G. Chen, and L. Bushnell, "Distributed observers design for leader-following control of multi-agent networks," Automatica, vol. 44, no. 3, pp. 846-850, 2008.

[12] Z. Li, X. Liu, P. Lin et al., "Consensus of linear multi-agent systems with reduced-order observer-based protocols," Systems \& Control Letters, vol. 60, pp. 510-516, 2011.

[13] H. Zhang, G. Feng, H. Yan, and Q. Chen, "Observer-based output feedback event-triggered control for consensus of multiagent systems," IEEE Transactions on Industrial Electronics, vol. 61, no. 9, pp. 4885-4894, 2014.

[14] H. Kim, H. Shim, and J. H. Seo, "Output consensus of heterogeneous uncertain linear multi-agent systems," IEEE Transactions on Automatic Control, vol. 56, no. 1, pp. 200-206, 2011.

[15] T. Liu and Z.-P. Jiang, "Distributed output-feedback control of nonlinear multi-agent systems," Institute of Electrical and Electronics Engineers Transactions on Automatic Control, vol. 58, no. 11, pp. 2912-2917, 2013.

[16] G. Guo, L. Ding, and Q.-L. Han, "A distributed event-triggered transmission strategy for sampled-data consensus of multiagent systems," Automatica, vol. 50, no. 5, pp. 1489-1496, 2014.

[17] Y. Hong, J. Hu, and L. Gao, "Tracking control for multiagent consensus with an active leader and variable topology," Automatica, vol. 42, no. 7, pp. 1177-1182, 2006.

[18] G. Wen, Z. Duan, G. Chen, and W. Yu, "Consensus tracking of multi-agent systems with Lipschitz-type node dynamics and switching topologies," IEEE Transactions on Circuits and Systems I: Regular Papers, vol. 61, no. 2, pp. 499-511, 2014.

[19] Y. Zhao, Z. Duan, and G. Wen, "Distributed finite-time tracking control for multi-agent systems: an observer-based approach," Systems \& Control Letters, vol. 62, no. 1, pp. 22-28, 2013.

[20] S. Mastellone, D. M. Stipanović, C. R. Graunke, K. A. Intlekofer, and M. W. Spong, "Formation Control and Collision Avoidance for Multi-agent Non-holonomic Systems: Theory and Experiments," International Journal of Robotics Research, vol. 27, no. 1, pp. 107-126, 2008.

[21] Z. Y. Lin, L. L. Wang, Z. M. Han, and M. Y. Fu, "Distributed formation control of multi-agent systems using complex laplacian," IEEE Transactions on Automatic Control, vol. 59, no. 7, pp. 1765-1777, 2014.

[22] H. Shi, L. Wang, and T. Chu, "Swarming behavior of multi-agent systems," Control Theory and Technology, vol. 2, no. 4, pp. 313318, 2004

[23] Y. Dong and J. Huang, "A leader-following rendezvous problem of double integrator multi-agent systems," Automatica, vol. 49, no. 5, pp. 1386-1391, 2013.

[24] F. Xiao, L. Wang, J. Chen, and Y. Gao, "Finite-time formation control for multi-agent systems," Automatica, vol. 45, no. 11, pp. 2605-2611, 2009.
[25] C.-L. Liu and Y.-P. Tian, "Formation control of multi-agent systems with heterogeneous communication delays," International Journal of Systems Science, vol. 40, no. 6, pp. 627-636, 2009.

[26] L. Consolini, F. Morbidi, D. Prattichizzo, and M. Tosques, "Leader-follower formation control of nonholonomic mobile robots with input constraints," Automatica, vol. 44, no. 5, pp. 1343-1349, 2008.

[27] Z. Peng, G. Wen, A. Rahmani, and Y. Yu, "Leader-follower formation control of nonholonomic mobile robots based on a bioinspired neurodynamic based approach," Robotics and Autonomous Systems, vol. 61, no. 9, pp. 988-996, 2013.

[28] W. Yu, G. Chen, and J. Lv, "On pinning synchronization of complex dynamical networks," Automatica, vol. 45, no. 2, pp. 429-435, 2009.

[29] C. Godsil and G. Royle, Algebraic Graph Theory, Springer, New York, NY, USA, 2001.

[30] T. Chen, X. Liu, and W. Lu, "Pinning complex networks by a single controller," IEEE Transactions on Circuits and Systems I: Regular Papers, vol. 54, no. 6, pp. 1317-1326, 2007. 


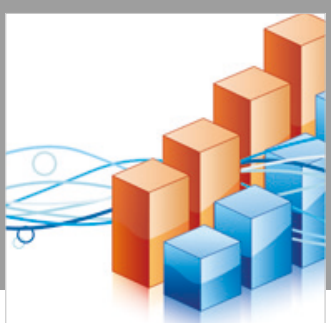

Advances in

Operations Research

\section{-n-m}
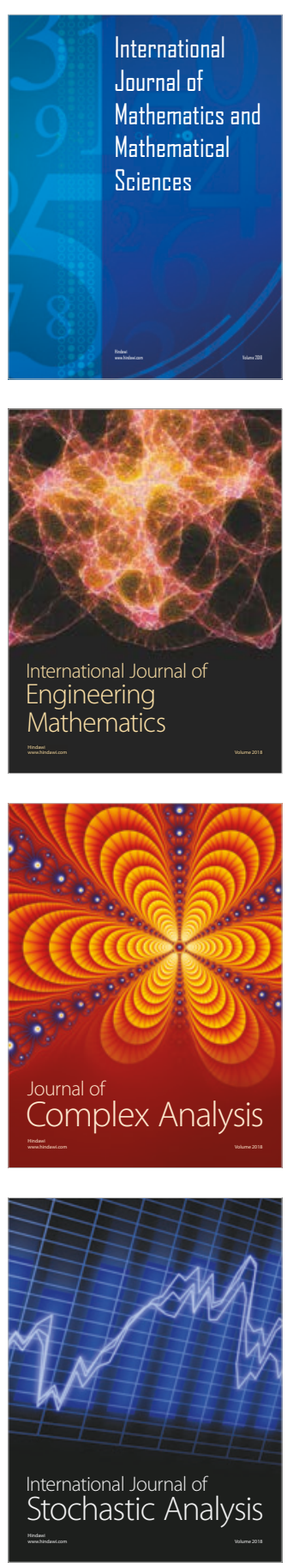
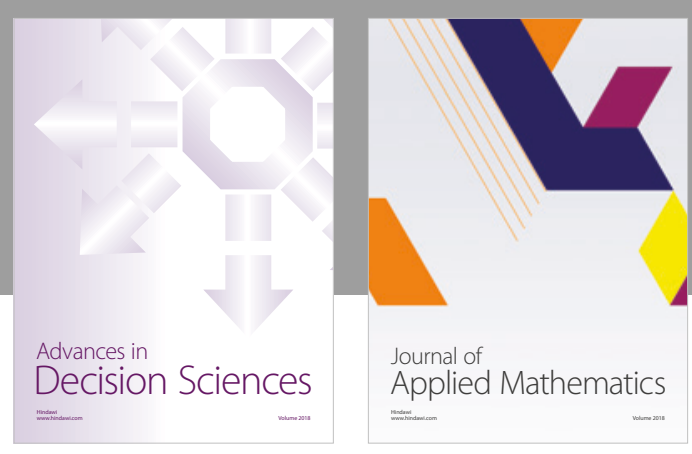

Journal of

Applied Mathematics
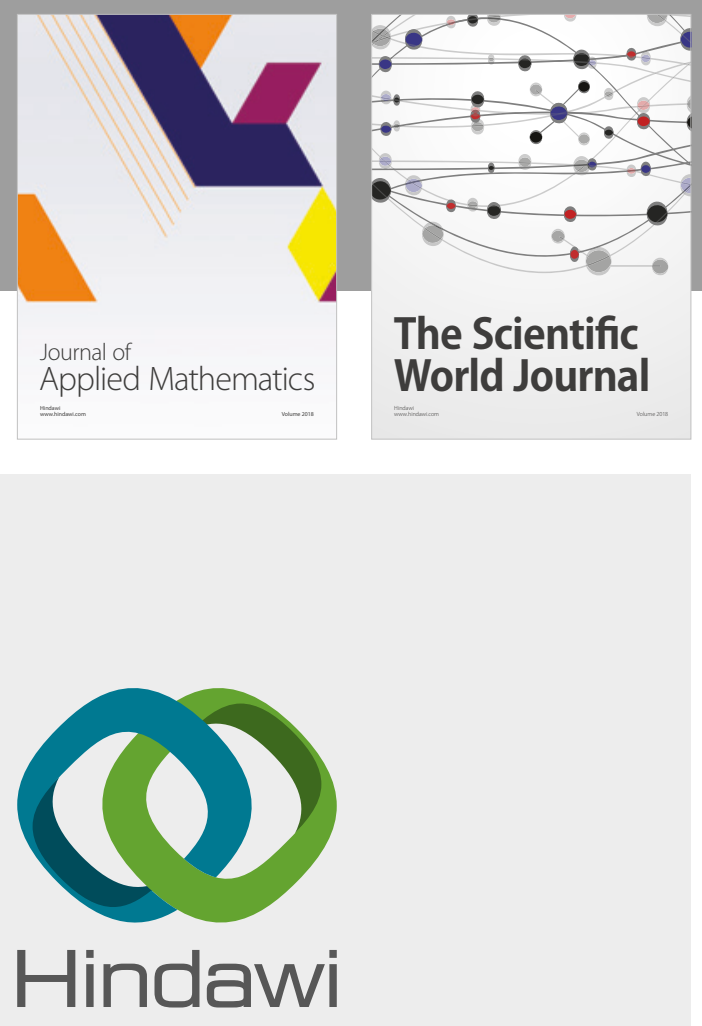

Submit your manuscripts at

www.hindawi.com

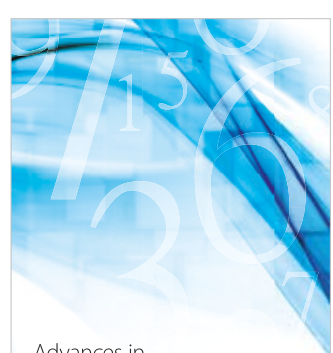

Advances in
Numerical Analysis
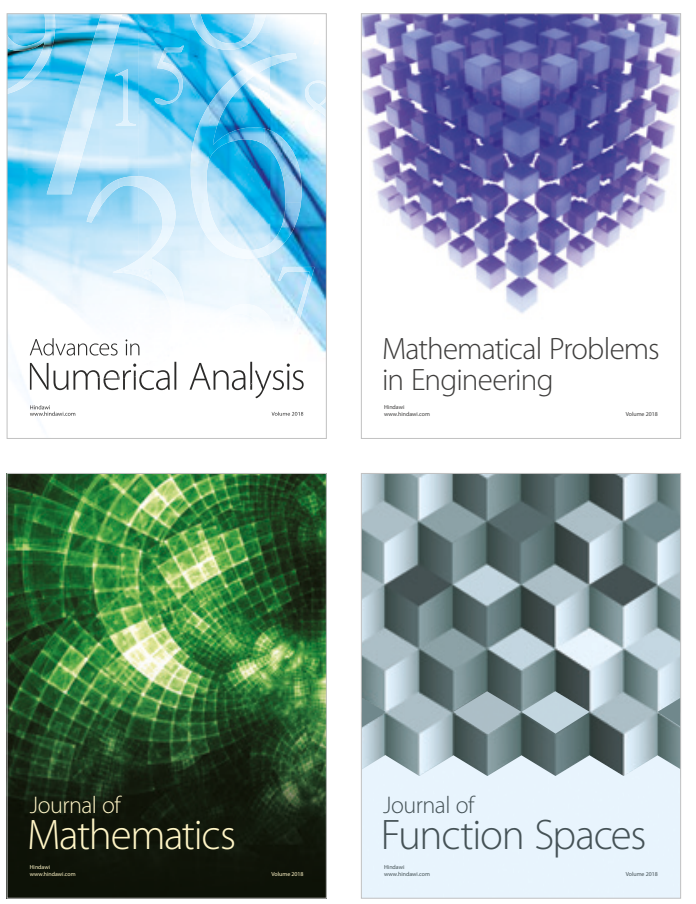

Mathematical Problems in Engineering

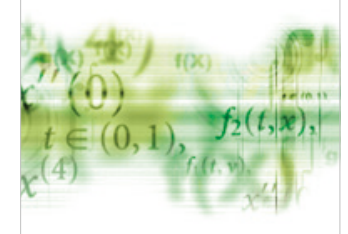

International Journal of

Differential Equations

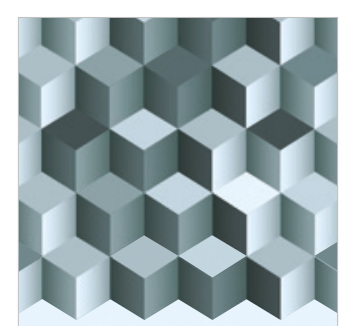

Journal of

Function Spaces

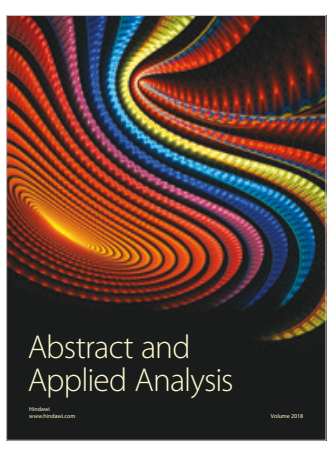

The Scientific

World Journal

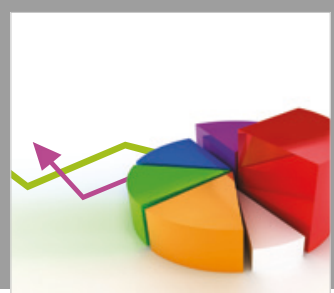

Journal of

Probability and Statistics
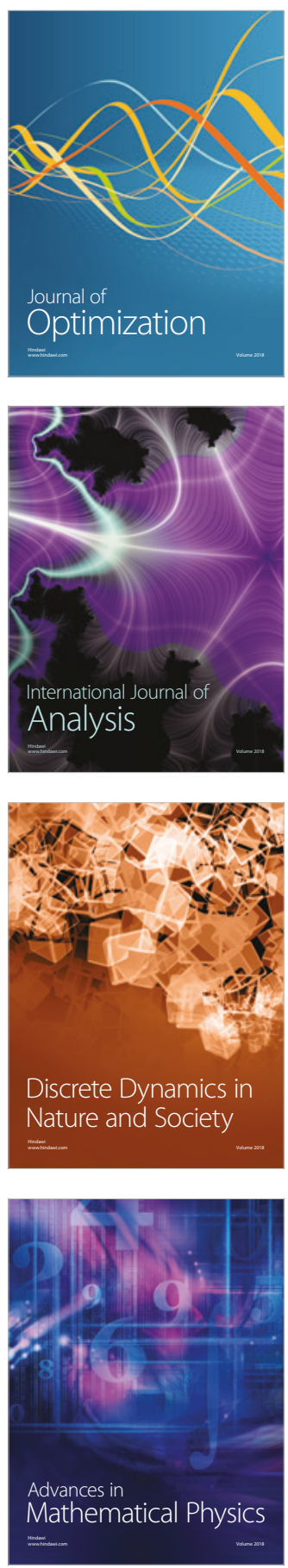